\title{
H5N1 Protection by Seasonal Influenza Vaccine in Homologous and Heterologous Prime/Boost Vaccination
}

Roos A ${ }^{1}$, Roozendaal $\mathbf{R}^{1^{*}}$, Damman-Riahi $\mathbf{S}^{1}$, Vreugdenhil $\mathbf{J}^{1}$, Vaneman $\mathbf{J}^{1}$, Dekking $\mathbf{L}^{1}$, Koldijk $\mathbf{M}^{2}$, Goudsmit $\mathbf{J}^{2}$ and Radošević $\mathbf{K}^{3}$

${ }^{1}$ Infectious Diseases and Vaccines Therapeutic area, Janssen Research and Development, Pharmaceutical companies of Johnson and Johnson, Leiden, The Netherlands

${ }^{2}$ Janssen Prevention Center, Center of Excellence within Janssen Research \& Development, Janssen, Pharmaceutical companies of Johnson and Johnson, Leiden, The Netherlands

${ }^{3}$ Sanofi, Global Biotherapeutics, Vitry-sur-Seine, France

"Corresponding author: Roozendaal R, Archimedesweg 4, 2333CN Leiden, The Netherlands; Tel: +31-71-5199123; E-mail: RRoozend@its.jnj.com

Received date: Sep 23, 2015; Accepted date: Nov 18, 2015; Published date: Nov 21, 2015

Copyright: (c) 2015 Roos A, et al. This is an open-access article distributed under the terms of the Creative Commons Attribution License, which permits unrestricted use, distribution, and reproduction in any medium, provided the original author and source are credited.

\begin{abstract}
Background

A number of different approaches aimed at broadening the cross-protective ability of seasonal influenza vaccines are being explored today. Priming a seasonal vaccine with three administrations of DNA encoding H1 HA corresponding to the HA in the seasonal vaccine has been shown to confer protection against heterologous H1N1 influenza. Here we evaluated the heterosubtypic protection induced by a seasonal influenza vaccine when primed with H1 HA DNA and, in parallel, when given as a homologous prime/boost regimen.

\section{Methods}

Balb/c mice were immunized three times with vaccine homologous H1 HA DNA prior to a boost with seasonal influenza vaccine (season 2009/2010; Northern Hemisphere), or immunized three times with the seasonal influenza vaccine. To assess cross-protection, mice were subsequently challenged with either heterologous H1N1 or heterosubtypic H5N1 influenza virus.

\section{Results}

The level of heterologous H1N1 protection elicited by the seasonal influenza vaccine was enhanced by priming with H1 HA DNA. In contrast, priming with H1 HA DNA did not enhance the level of heterosubtypic H5N1 protection. The heterologous prime boost regimen showed to be less efficient than multiple immunizations with seasonal vaccine in conferring protection against H5N1. Neither the DNA-priming vaccination regimen, nor the homologous prime/boost regimen induced detectable $\mathrm{H} 5 \mathrm{~N} 1$ cross-reactive anti-HA or anti-NA antibodies. Homologous prime /boost vaccination did induce higher levels of anti-NP antibodies.

\section{Conclusion}

Here we demonstrate that priming a seasonal influenza vaccine with vaccine homologous $\mathrm{H} 1 \mathrm{HA}$ encoding DNA enhances the level of heterologous H1N1 but not heterosubtypic protection induced by the vaccine alone. Homologous prime/boost vaccination resulted in higher levels of heterosubtypic protection. Of the immunogenicity parameters tested for both heterologous and homologous prime/boost regimens only anti-NP responses follow the same pattern as heterosubtypic protection.
\end{abstract}

Keywords: Influenza; Seasonal influenza vaccine; prime boost; HA DNA; Protection; H5N1; NP

\section{Introduction}

Annual influenza epidemics are responsible for up to 5 million cases of severe illness and 250000 to 500000 deaths as reported by the world health organization (WHO) [1]. Seasonal influenza vaccines are the most effective way to reduce the impact of influenza epidemics. To keep pace with the genetically drifted circulating strains the composition of seasonal influenza vaccines must be updated almost annually [2]. The development of an influenza vaccine which confers protection against a wide range of influenza viruses is therefore of great importance.

The efforts to generate broadly protective influenza vaccines have largely focused on designing vaccine compositions and regimens able to induce immune responses, either humoral, cellular or a combination thereof, against proteins that are conserved across divergent strains of influenza [3,4]. Novel influenza vaccines that aim to elicit crossprotective T-cell responses are often based on highly conserved viral 
Citation: Roos A, Roozendaal R, Riahi SD, Vreugdenhil J, Vaneman J, et al. (2015) H5N1 Protection by Seasonal Influenza Vaccine in Homologous and Heterologous Prime/Boost Vaccination. J Vaccines Vaccin 6: 299. doi:2157-7560.1000299

Page 2 of 9

proteins such as nucleoprotein (NP) [5-7] and matrix protein 1 (M1) [8], while vaccines that aim to induce broad humoral immunity have largely focused on surface exposed viral proteins such as hemagglutinin (HA) [9-13], neuraminidase (NA) [14,15] or matrix protein 2 (M2) [16-18]. Though HA and NA are highly variable proteins, they contain conserved epitopes that can elicit broadly protective antibodies [19-24], and as such are very interesting targets in the quest for universal influenza vaccines. B cells producing HAspecific broadly neutralizing antibodies (bnAbs) have been isolated from human volunteers after vaccination with seasonal vaccines $[21,25,26]$, indicating that HA in seasonal vaccines can form the basis for developing a broadly protective immune response. While HA is the key immunogen in all current seasonal vaccines, and is used for vaccine dosing and evaluation of the seasonal vaccine potency, the majority of current vaccines also contain other viral proteins, such as NA, M2 and NP [27,28], albeit amounts thereof are neither quantified nor standardized. The immune response to these proteins may potentially contribute to cross-protective ability of seasonal influenza vaccines.

Different approaches aimed at broadening cross-protective ability of seasonal influenza vaccines are being explored [29-31]. We reported previously [32] that multiple immunizations with seasonal influenza vaccine of season 2011-2012 can enhance the heterosubtypic (H5N1) protection in mice. In other approaches, seasonal influenza vaccine has been administered together with adjuvants $[31,33,34]$ or primed with DNA vectors [30]. Wei et al. [30] demonstrated that heterologous H1N1 protection can be induced in both mice and ferrets by priming a seasonal vaccine with three administrations of DNA encoding H1 HA corresponding to the $\mathrm{H} 1$ present in the seasonal influenza vaccine $(\mathrm{H} 1-$ DNA). The safety and efficacy of this vaccination regimen, expanded to include DNAs encoding $\mathrm{H} 3$ and $\mathrm{B}$ HA, is being further explored in healthy human volunteers [35].

In the current study, we expanded the evaluation of a heterologous prime/boost vaccination regimen (H1-DNA, followed by seasonal influenza vaccine) to induce heterosubtypic protection against $\mathrm{H} 5 \mathrm{~N} 1$. In parallel, we assessed the breadth of protection and immunogenicity induced by a seasonal influenza vaccine of season 2009/2010 when given in a homologous (e.g., $3 \mathrm{x}$ seasonal vaccine) [32] or heterologous prime/boost vaccination regimen.

\section{Material and Methods}

\section{Statement of ethics}

All mouse experiments were performed in accordance with Dutch legislation on animal experiments and approved by DEC Animal Sciences Group, Wageningen UR when performed at CVI Lelystad and an independent Animal Ethics Committee (TNO, Zeist, The Netherlands) (permit number 3387) when performed at TNO Triskelion. In all experiments six-to-eight-week-old female Balb/c (H2d) mice (Charles River, Sulzfeld, Germany) were used. Mice were kept under specific pathogen-free conditions.

\section{Immunization and Influenza Challenge}

\section{Immunization}

Groups of mice ( $n=10$ or 8 for challenge studies, $n=8$ for immunogenicity study) received either: $1 \mathrm{x}$ or $3 \times$ intramuscular (i.m.) immunizations with Inflexal V (Crucell, Bern, Switzerland), a trivalent seasonal vaccine (SV) (composition for the 2009-2010 season: H1N1 A/Brisbane/59/07, H3N2 A/Brisbane/10/07 and B/Brisbane/60/08) (3 $\mu \mathrm{g}$ HA per strain per immunization); $3 \mathrm{x}$ i.m. immunizations with DNA encoding RSV_F_A2 protein (15 $\mu$ g per immunization) followed by an i.m. immunization with SV; $3 \times$ i.m. immunizations with DNA encoding HA of $\mathrm{H} 1 \mathrm{~A} /$ Brisbane/59/07 (15 $\mu \mathrm{g}$ per immunization); $3 \times$ i.m., immunizations with DNA encoding HA of H1 A/Brisbane/59/07 (15 $\mu$ g per immunization) followed by one i.m., immunization with SV; or $4 \times$ with PBS (Gibco, Life TechnologiesTM, Paisley, UK). Immunizations were scheduled at 3 week intervals. All final immunizations with vaccine were performed at the same time. Mice immunized only once with SV, received three immunizations with PBS prior to vaccine. Mice immunized either $3 \times$ with SV or $3 x$ with DNA encoding H1 HA A/Brisbane/59/07 received one immunization with PBS prior to vaccine.

\begin{tabular}{|c|c|c|c|c|c|}
\hline \multirow{2}{*}{$\begin{array}{l}\text { Description } \\
\text { Vaccine group }\end{array}$} & \multicolumn{5}{|l|}{ Day } \\
\hline & 0 & 21 & 42 & 63 & $91^{\mathrm{d}, \mathrm{e}}$ \\
\hline PBS & PBS & PBS & PBS & PBS & Challenge \\
\hline Sv09 & & & & SV09c & Challenge \\
\hline 3xSV09 & & SV09c & Sv09c & SV09c & Challenge \\
\hline H1-DNA & & $\begin{array}{l}\text { H1- } \\
\text { DNA }^{b}\end{array}$ & $\begin{array}{l}\text { H1- } \\
\text { DNA }\end{array}$ & $\begin{array}{l}\text { H1- } \\
\text { DNA }^{b}\end{array}$ & Challenge \\
\hline RSV-DNA/SV09 & $\begin{array}{l}\text { RSV- } \\
\text { DNA }^{a}\end{array}$ & $\begin{array}{l}\text { RSV- } \\
\text { DNA }^{a}\end{array}$ & $\begin{array}{l}\text { RSV- } \\
\text { DNA }^{a}\end{array}$ & SV09c & Challenge \\
\hline H1-DNA/SV09 & $\mathrm{H} 1-\mathrm{DNA} \mathrm{A}^{\mathrm{b}}$ & $\begin{array}{l}\mathrm{H} 1- \\
\mathrm{DNA}^{\mathrm{b}}\end{array}$ & $\begin{array}{l}\text { H1- } \\
\text { DNA }^{b}\end{array}$ & SV09c & Challenge \\
\hline
\end{tabular}

aRSV-DNA: pcDNA2004(Neo-) containing RSV-F-A2 cDNA insert. Dose: $15 \mu \mathrm{g}$ i.m./immunization

bH1-DNA: pcDNA2004(Neo-) containing H1 A/Brisbane/59/07 cDNA insert. Dose: $15 \mu \mathrm{g}$ i.m./immunization

CSV09: Seasonal Influenza vaccine, Inflexal® V, of season 2009-2010. Dose 3 $\mu \mathrm{g} \mathrm{HA} /$ strain/ immunization

${ }^{d}$ For immunogenicity experiments mice were sacrifised at day 91 and serum and spleens were collected. For influenza

challenge experiments mice were challenged at day 91

eChallenge: 25xLD50 of H1N1A/Puerto Rico/8/34, H1N1 A/WSN/33 or H5N1 A/ Hong Kong/156/97

Table 1: A schematic overview of the immunization regimens for the various vaccine groups is presented.

In immunogenicity experiments blood and spleens were harvested four weeks after final immunization. Blood was collected via heart puncture under isoflurane anesthesia (IsoFlo", Abbott Park, IL, USA) followed by cervical dislocation and collection of the spleen. Serum was collected after centrifugation for 4 minutes at $1699 \times \mathrm{g}$ followed by 1 minute at $20817 \times \mathrm{g}$. The serum was isolated and stored at $-20^{\circ} \mathrm{C}$.

\section{Influenza challenge}

For challenge experiments mice were infected with influenza virus 4 weeks after the final immunization. On the day of challenge a prechallenge blood sample (to assess pre-challenge antibody titers) was obtained via submandibular bleeding. Mice were challenged intranasally (i.n.) with $25 \times$ LD50 of influenza virus (total $50 \mu \mathrm{l}, 25 \mu \mathrm{l}$ per nostril) under anesthesia with ketamine/xylazine $(100 \mathrm{mg} / \mathrm{kg}$ ketamine (Nimatek $100 \mathrm{mg} / \mathrm{ml}$, Eurovet, Cuijk, the Netherlands); 20 $\mathrm{mg} / \mathrm{kg}$ xylazine (Sedamun $20 \mathrm{mg} / \mathrm{ml}$, Eurovet, Cuijk, Netherlands)). 
Virus stocks of H1N1 A/Puerto Rico/8/34 (ATCC-VR-95, American Type Culture Collection, Manassas, VA, USA), H1N1 A/WSN/33 (Central Veterinary Institute, Wageningen University, the Netherlands) and wild-type H5N1 A/Hong Kong/156/97 (Central Veterinary Institute, Wageningen University, the Netherlands) were grown on embryonated chicken eggs. Groups of mice receiving $4 \times$ PBS i.m., were used as negative control and groups receiving broadly protective monoclonal antibody (CR6261, $15 \mathrm{mg} / \mathrm{kg}$ in PBS intravenously (i.v.) 24 hours prior to challenge) were used as positive control for the challenge. After challenge mice were monitored daily for weight-loss and clinical score for up to 21 days or until a humane endpoint based on clinical score or found dead. The challenge experiments were performed at two different locations at which different clinical score systems were used. The H1N1 A/WSN/33 and H5N1 A/Hong Kong/ $156 / 97$ challenge studies were performed at CVI Lelystadt at which a 4 -point clinical scoring system was used: $0=$ no clinical signs, $1=$ rough coat, $2=$ rough coat, less reactive, passive during handling, $3=$ rough coat, rolled up, labored breathing, passive during handling, $4=$ rough coat, rolled up, labored breathing, unresponsive. CS4 was defined as moribund based on unresponsiveness and used as a humane endpoint. The H1N1 A/Puerto Rico/8/34 challenge study was performed at TNO Triskelion at which a 5 -point clinical scoring system was used: $0=$ no clinical signs, $1=$ rough coat, $2=$ rough coat, labored respiration $3=$ rough coat, labored respiration, hunched posture and/or blepharospasm, 4=rough coat, labored respiration, hunched posture, blepharospasm, lethargic and/or thin/dehydrated, 5=lethargic behavior (CS4) is observed during four consecutive observations leading to euthanasia. CS5 was used as humane endpoint.

\section{Statistics}

\section{Influenza challenge studies}

Differences between immunization regimens relative to negative control group receiving $4 \times$ PBS i.m., were statistically evaluated using survival proportion, survival time, change in bodyweight and clinical scores. Survival proportion and survival time after challenge were analyzed using Fisher's exact test and log-rank test, respectively. Repeated measurements in the challenge phase (i.e. bodyweight and clinical scores) were summarized as a single outcome per animal using an Area Under the Curve (AUC) approach where missing values for animals that died early were imputed with a last-observation-carriedforward method. Body weight data are expressed as the change relative to the day 0 measurement. The AUC was then defined as the summation of the area above and below the baseline. An ANOVA on AUC's was done with group as explanatory factor. Clinical scores were summarized as AUC per mouse and groups were compared using a generalized linear model with a cumulative logit distribution to compare area under the curves for ordinal variable. Statistical analysis was planned upfront and adjustments for multiple comparisons were done using a Bonferroni correction for (i) the H1N1 A/Puerto Rico/ 8/34 challenge: $\mathrm{H} 1-\mathrm{DNA} / \mathrm{SV}=4$ comparisons, $\mathrm{SV}=2$ comparisons (clinical score data for this group were not adjusted), (ii) the $\mathrm{H} 1 \mathrm{~N} 1$ A/WSN/33 challenge: for all groups 4 comparisons, (iii) figure 1C H5N1 A/Hong Kong/156/97 challenge: H1-DNA/SV=4 comparisons, $\mathrm{SV}=2$ comparisons (clinical score data for this group were not adjusted), (iv) figure 2 H5N1 A/Hong Kong/156/97 challenge: for all groups 2 comparisons. The studies were considered valid only when there was a statistically significant difference in survival proportion (Fisher's exact-test, 2-sided) between negative and positive challenge model control groups (data not shown for positive controls).
Statistical analyses were performed using SAS version 9.2 (SAS Institute Inc. Cary, NC, USA) and SPSS version 20 (IBM, USA). Statistical tests were conducted two-sided at an overall significance level of $\alpha=0.05$. Only $p$ values less than 0.05 are reported in the Result section. A summary of all statistical tests and respective $\mathrm{p}$ values are presented in supplementary Table S1.

\section{Virus neutralization assay}

Madin-darby canine kidney (MDCK) cells were seeded in a 96-well plate at 15,000 cells/well in growth medium (Dulbecco's Modified Eagle Medium (DMEM) containing $200 \mathrm{mM} \mathrm{L}$-glutamine, $3 \mu \mathrm{g} / \mathrm{ml}$ trypsin and $1 \%(\mathrm{w} / \mathrm{v})$ penicillin/streptomycin stock solution, all Gibco, Invitrogen Ltd, Life Technologies, Paisley, UK) and allowed to attach for a minimum of 3 hours. Duplicate serial dilutions of heatinactivated $\left(30\right.$ minutes at $\left.56^{\circ} \mathrm{C}\right)$ serum samples $(0.01-20 \%)$ were prepared in DMEM with or without trypsin/EDTA $(0.6 \%$ of a $0.05 \%$ stock solution) and mixed with 120 TCID50 of H1N1 A/Brisbane/ $59 / 07$ or 200 TCID50 of the H5N1 A/Hong Kong/156/97 (reassortant rgPR8-H5N1) virus per sample, respectively, for 1 hour at $37^{\circ} \mathrm{C}, 10 \%$ $\mathrm{CO}_{2}$. Mixes were subsequently added to the MDCK cells and incubated for 18 hours at $37^{\circ} \mathrm{C}, 10 \% \mathrm{CO}_{2}$. Cells were fixed with $80 \%$ acetone, labeled with mouse anti-NP (H16-L10-4R5, produced inhouse), followed by goat anti-mouse HRP-coupled antibody (KPL, Gaithersberg, MD, USA) for one hour each. TMB substrate (Roche, Basel, Switzerland) was added, and absorbance was read in a BioTek reader (PerkinElmer, Groningen, the Netherlands) after 5-15 minutes. Monoclonal antibody CR6261 (human IgG2a, produced in-house) and naïve mouse serum were used as positive and negative controls, respectively. Samples without detectable neutralization at the lowest dilution are indicated as the lowest dilution (i.e. background level). The IC50 values were calculated after 4-parameter logistic curve fit.

\section{T-cell ELISPOT}

For ELISPOT analysis of T-cell responses ten amino acids overlapping 15-mer peptides covering the whole HA protein sequence of H1 A/Brisbane/59/07, a total of 104 peptides, were used (Pepscan, Lelystad, The Netherlands). For analysis of the total T-cell response against the full length $\mathrm{H} 1 \mathrm{~A} /$ Brisbane/59/07 a pool of all 111 peptides was made (total pool). For analysis of T-cell response against a known 9-mer epitope IYSTVASSL [36], highly conserved among number of strains, including H1 A/Brisbane/59/07 and H5 A/Hong Kong/156/97, two 15-mer peptides containing this epitope were used. The concentration per peptide was $0.4 \mathrm{mg} / \mathrm{ml}$, diluted in DMSO.

Ninety-six-well multiscreen plates (Millipore, Bedford, MA), coated overnight with rat anti-mouse IFN $\gamma$ (Pharmingen, San Diego, CA) (1 $\mu \mathrm{g}$ per well in PBS pH 7.4), were washed with Dulbecco's PBS (Life Technologies, Gaithersberg, MD) containing 0.05\% Tween-20 (D-PBS/ Tween) and blocked with D-PBS containing 5\% FBS for 2 hours at $37^{\circ} \mathrm{C}$. Splenocytes were prepared in R10 medium (RPMI 1640 (Gibco/ Invitrogen, Breda, The Netherlands) containing $10 \%$ heat inactivated FBS (HyClone, Logan UT), 1\% Pen/Strep (Gibco/Invitrogen, Breda, The Netherlands), 1\% MEM non-essential amino acids (Gibco/ Invitrogen, Breda, The Netherlands) and $13 \mu \mathrm{M}$ 2-mercaptoethanol (Fluka Chemie, Buchs, Switzerland) and plated in duplicates at $5 \mu 10^{5}$ cells/well and $2 \times 10^{5}$ cells/well in a $100-\mu l$ reaction volume containing $2 \mu \mathrm{g} / \mathrm{ml} 15$-mer peptides (total pool or two 15-mer peptides). Following 18 hour incubation at $37^{\circ} \mathrm{C}$, the plates were washed with $\mathrm{D}$ $\mathrm{PBS} /$ Tween and incubated for 1.5 hour with a biotinylated rat antimouse IFN $\gamma$ (Pharmingen, San Diego, CA). Plates were washed and 
Citation: Roos A, Roozendaal R, Riahi SD, Vreugdenhil J, Vaneman J, et al. (2015) H5N1 Protection by Seasonal Influenza Vaccine in Homologous and Heterologous Prime/Boost Vaccination. J Vaccines Vaccin 6: 299. doi:2157-7560.1000299

Page 4 of 9

incubated for 1.5 hour with streptavidin-alkaline phosphatase (Southern Biotechnology Associates, Birmingham, AL). Upon final washing, specific staining was developed with nitro blue tetrazolium-5bromo-4-chloro-3-indolyl-phosphate chromogen (Pierce, Rockford, IL), stopped by washing with tap water, air dried, and analyzed using an AELVIS ELISPOT reader (AELVIS GmbH). The cell concentration for which the average of spot forming units (SFU)/well was within the linear range, 50-225 spots/well, was selected. The average SFU/well count was adjusted to SFU's per $10^{6}$ cells in accordance with cell dilution.

\section{ELISA}

Recombinant protein $(0.05 \mu$ g per well in PBS pH7.4) (i) HA of H1N1 A/Brisbane/59/07 (Protein Sciences Inc., CT, USA), H1N1 A/ Puerto Rico/8/34 (Protein Sciences Inc., CT, USA) or of H5N1 A/Hong Kong/156/97 (manufactured in-house on HEK293F cells), (ii) NP of H1N1 A/Puerto Rico/8/34 (Sino Biologics Inc. Beijing, China), or (iii) NA of H1N1 A/Brisbane/59/07 (manufactured in-house on HEK293F cells) or of H5N1 A/Hong Kong/156/97 (manufactured in-house on HEK293F cells) were coated onto Maxisorp 96-well plates (Nunc", Thermo Scientific) $\mathrm{O} / \mathrm{N}$ at $4^{\circ} \mathrm{C}$. Plates were washed with PBS (Gibco, Life Technologies ${ }^{\text {tw }}$, Paisley, UK) containing 0.05\% Tween-20 (Calbiochem ', Merck Millipore, Darmstadt, Germany) (PBS-T) and subsequently blocked with for rHA and rNP ELISA; PBS containing $2 \%$ dried skimmed milk (Difco "', BD, Breda, the Netherlands) and for rNA ELISA; PBS containing 2\% BSA (Sigma-Aldrich, USA) for 1 hour at RT. Following a wash with PBS-T serum was added to the plate. The serum was in duplicate serially diluted (2-fold, 0.002-2\%) and incubated for 1 hour at RT. Following a wash with PBS-T a 1:2000 dilution of Goat-anti-Mouse IgG-HRP (KPL, Gaithersburg, MD, USA) was added to the plate and incubated for 1 hour at RT. After washing with PBS-T OPD substrate (Thermo Scientific, Bremen, Germany) was added to the plate. The colorimetric reaction was stopped after 10 minutes by adding $1 \mathrm{M} \mathrm{H}_{2} \mathrm{SO}_{4}$. The optical density (OD) was measured at $492 \mathrm{~nm}$ and standard curves were created using a four parameter logistic curve. The OD of each sample dilution was then quantified against the standard curve and the final concentration per sample (in Elisa Units, EU/ml) calculated by a weighted average, using the squared slope of the standard curve at the location of each quantification as weight. Negative samples were set at the limit of detection (LOD), defined as the lowest sample dilution multiplied by the lowest standard concentration with an OD response above the lower asymptote of the standard curve and background. All ELISA titers presented in the figures have been $\log 10$ transformed.

\section{Results}

Priming seasonal influenza vaccine with H1-DNA enhances protection against heterologous $\mathrm{H} 1 \mathrm{~N} 1$ but not against heterosubtypic H5N1 challenge:

We first tested whether a virosomal seasonal influenza vaccine of season 2009/2010 (SV09) primed three times with DNA encoding vaccine homologous $\mathrm{H} 1 \mathrm{HA}$ (H1-DNA) can provide heterologous H1N1 protection in mice. Albeit not significant, a single immunization with SV09 alone elicited partial survival (40\%) against heterologous H1N1 A/Puerto Rico/8/34 with significantly prolonged survival time ( $\mathrm{p}=0.003$ compared to PBS) and with reduced clinical scores $(\mathrm{p}=0.001$ compared to PBS). When primed with H1-DNA, SV09 elicited a significant increase in survival proportion compared to nonvaccinated mice ( $60 \%$ survival, $\mathrm{p}=0.043$ compared to $\mathrm{PBS})$ with prolonged survival time ( $\mathrm{p}=0.044$ compared to $\mathrm{PBS}$ ), reduced bodyweight loss ( $\mathrm{p}=0.050$ compared to $\mathrm{PBS}$ ) and reduced clinical scores ( $<<0.001$ compared to PBS) (Figure 1A and S1.A).

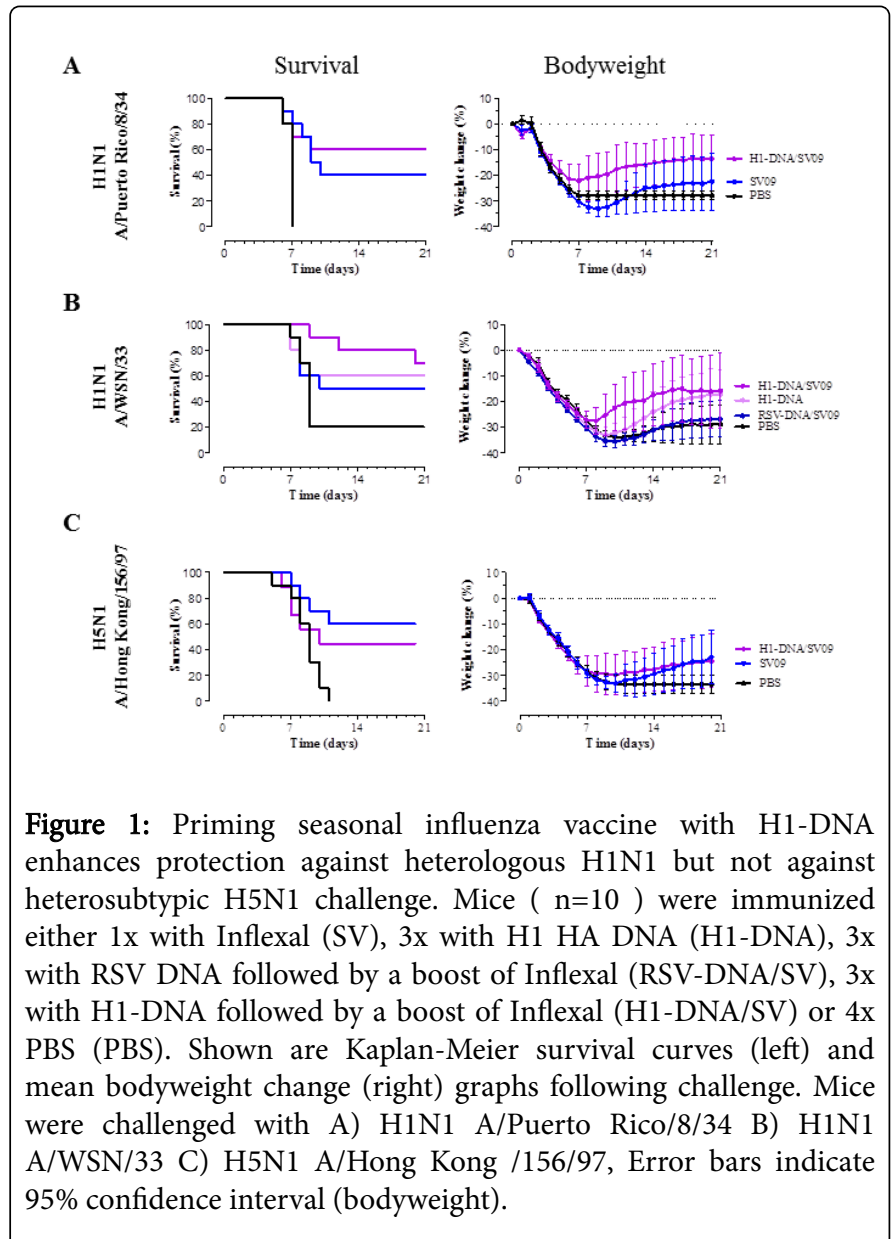

To confirm the effect of DNA priming in another heterologous challenge model, we assessed protection by H1-DNA/SV09 upon challenge with $\mathrm{H} 1 \mathrm{~N} 1 \mathrm{~A} / \mathrm{WSN} / 33$. Two additional groups were included in this experiment to further delineate the contribution of H1-DNA to protection: one group of mice was immunized with H1-DNA only and another group three times with DNA encoding an irrelevant antigen (RSV_F_A2 protein) (RSV-DNA) followed by a boost of SV09 (RSVDNA/SV09). H1-DNA/SV09 induced 70\% survival after H1N1 A/WSN/33 challenge (not statistically significant), with prolonged survival time ( $\mathrm{p}=0.038$ compared to $\mathrm{PBS}$ ) and reduced clinical scores $(\mathrm{p}=0.001$ compared to PBS) (Figure $1 \mathrm{~B}$ and S1.B), confirming protective ability of the heterologous prime/boost vaccination regimen. Partial survival seen in groups immunized with H1-DNA or RSVDNA/SV, $60 \%$ and 50\% respectively, was neither significant in survival proportion or reduction of disease symptoms. Thus, the improved level of cross-protection achieved with the heterologous prime/boost regimen requires specific antigen-expressing DNA as a prime and is not due to possible non-specific effect of DNA administration. The level of heterologous H1N1 cross-protection induced by the H1-DNA/ SV09 vaccination regimen was comparable to the cross-protection induced by three vaccinations with SV09 (3xSV09) (Figure S2), a regimen previously shown to be broadly protective [32]. 
Citation: Roos A, Roozendaal R, Riahi SD, Vreugdenhil J, Vaneman J, et al. (2015) H5N1 Protection by Seasonal Influenza Vaccine in Homologous and Heterologous Prime/Boost Vaccination. J Vaccines Vaccin 6: 299. doi:2157-7560.1000299

Page 5 of 9

To further evaluate whether the heterologous prime/boost vaccination regimen could elicit also heterosubtypic protection we challenged mice immunized with SV09 alone or H1-DNA/SV09 with H5N1 A/Hong Kong/156/97. Partial protection against H5N1 was elicited already by SV09 alone with a significant increase in survival proportion, survival time and reduced clinical scores compared to PBS-vaccinated mice ( $\mathrm{p}=0.022, \mathrm{p}=0.015$ and $\mathrm{p}=0.005$, respectively). Priming SV09 three times with H1-DNA did not improve the heterosubtypic cross protection induced by SV09. H1-DNA/SV09 induced partial survival, $44 \%$, albeit not statistically significant, with reduced clinical scores ( $p=0.007$ relative to PBS) (Figure 1C and S1.C).

H1-DNA/SV09 confers partial protection while three times SV09 protects against heterosubtypic $\mathrm{H} 5 \mathrm{~N} 1$ challenge:

A homologous prime/boost vaccination regimen in which a SV of season 2011-2012 was given 3 times was previously shown to elicit $80 \%$ survival against $\mathrm{H} 5 \mathrm{~N} 1$ challenge [32]. Here, we assessed the ability of the SV of season 2009 to induce protection against H5N1 when given in either a homologous or heterologous prime/boost immunization regimen.

Consistent with our previous experiment, the H1-DNA/SV09 vaccination regimen partially protected mice. The survival proportion of $43 \%$ was not significantly increased compared to PBS but clinical scores were reduced ( $\mathrm{p}=0.021$ compared to PBS). A homologous prime/boost regimen consisting of $3 \times \mathrm{SV} 09$, however, induced significant survival ( $80 \%, p=0.003$ compared to PBS) with prolonged survival time $(\mathrm{p}<0.001$ compared to $\mathrm{PBS})$, reduced bodyweight loss and clinical score $(\mathrm{p}=0.001$ and $\mathrm{p}<0.001$ compared to PBS, respectively) (Figure 2 and $\mathrm{S} 3$ ).
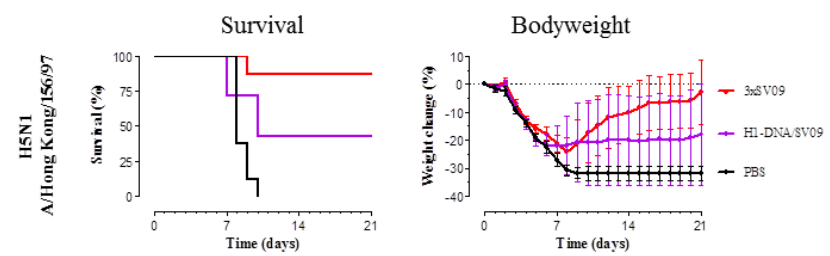

Figure 2: H1-DNA/SV09 confers only partial protection while three times SV09 protects against heterosubtypic H5N1 challenge. Mice were immunized either 3x with Inflexal 2009 (3xSV09), 3x with H1 HA DNA followed by a boost of Inflexal 2009 (H1-DNA/SV09) or $4 \mathrm{x}$ PBS (PBS) followed by challenge with H5N1 A/Hong Kong/ 156/97 ( $\mathrm{n}=8$ for PBS and 3x SV09; $\mathrm{n}=7$ for H1-DNA/SV09). Kaplan-Meier survival curves (left) and means bodyweight change (right). Error bars indicate 95\% confidence interval (bodyweight).

Neither neutralizing antibodies nor cross-reactive $\mathrm{T}$ cells reflect heterosubtypic protection induced by H1-DNA/SV09 and 3xSV09:

To elucidate possible mechanism of heterosubtypic protection, we characterized the neutralizing antibody and HA T-cell responses induced with H1-DNA/SV09 and $3 \times$ SV09 regimens against heterosubtypic (challenge) influenza strain (H5N1) and the SV09 H1N1 strain (A/Brisbane/59/07). Neither vaccination regimen elicited detectable cross-neutralizing antibodies, while both vaccination regimens induced high titers of vaccine homologous $\mathrm{H} 1 \mathrm{~N} 1$ neutralizing antibodies, with H1-DNA/SV09 regimen eliciting approximately 5.3 -fold higher mean titer compared to $3 \times$ SV09 (Figure $3 A)$. With respect to HA specific T-cell response, only the H1-DNA/
SV09 regimen elicited a T-cell response against both a highly conserved T-cell epitope in the stalk of the HA (IYSTVASSL, conserved in both $\mathrm{H} 1$ and $\mathrm{H} 5$ ) and the total pool of peptides from HA of A/Brisbane/59/07 (Figure 3B). Thus, neither cross-neutralizing antibodies nor HA-specific T-cell responses can explain the high heterosubtypic protection seen by $3 \times \mathrm{SV} 09$ versus suboptimal protection obtained with H1-DNA/SV09 vaccination regimen.

High titers of antibodies against NP, but not against H5 HA or NA, are elicited with both vaccination regimens and reflect observed difference in heterosubtypic protection:

We further characterized the humoral immune responses induced with the two vaccination regimens by measuring the total IgG response against HA, NA and NP in the pre-challenge serum. Pre-challenge antibody titers were used to assess the relationship between humoral immunogenicity and challenge outcome. Neither H1-DNA/SV09 nor 3xSV09 elicited significant rH5 A/Hong Kong/156/97 binding antibodies (except in 2 out of 8 mice from 3xSV09 group) (Figure 4A). Both regimens elicited high titer antibodies against vaccine $\mathrm{rH1}$ (A/ Brisbane/59/07).

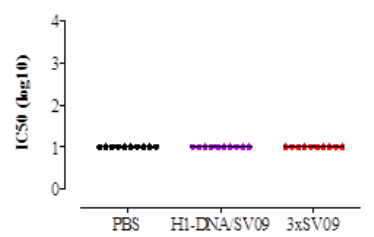

B

$$
\text { Conserved epitope }
$$
IYSTVASSL

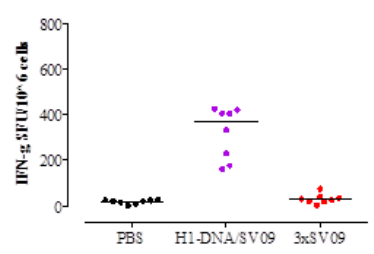

A H5N1 A/Hong Kong/156/97
H1N1 A/Brisbane/59/07

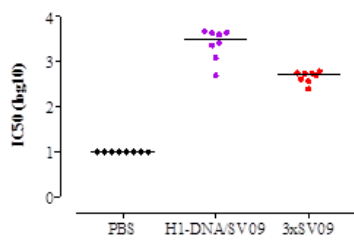

H1 A/Brisbane/59/07 15 -mer peptides

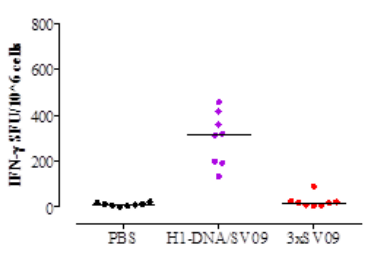

Figure 3: Neither neutralizing antibodies nor cross-reactive $\mathrm{T}$ cells reflect heterosubtypic protection induced by H1-DNA/SV09 and $3 x$ SV09. Characterization of humoral and cellular immune response induced by 3x Inflexal 2009 (3xSV09), 3x H1 HA DNA followed by a boost of Inflexal 2009 (H1-DNA/SV09) or 4x PBS (PBS), 4 weeks after final immunization $(\mathrm{n}=8)$. A) Neutralizing $\mathrm{Ab}$ titer against H5N1 A/Hong Kong/156/97 and H1N1 A/Brisbane/ 59/07. B) INF- $\gamma$ secreting T cells measured in ELISPOT against a pool of 215-mer peptides carrying conserved epitope; IYSTVASSL and a pool of 15-mer peptides of HA of H1N1 A/Brisbane/59/07. Group medians are shown.

The mean $\mathrm{rH} 1$ titer elicited with H1-DNA/SV09 was approximately 5.3-fold higher than the corresponding titer elicited with 3xSV09. Neither H1-DNA/SV09 nor 3xSV09 elicited significant rN1 A/Hong Kong/156/97 binding antibody titers (Figure 4B). A moderate titer of antibodies against vaccine $\mathrm{rN1}$ (A/Brisbane/59/07) was detected after 3xSV09, but not after H1-DNA/SV09. 


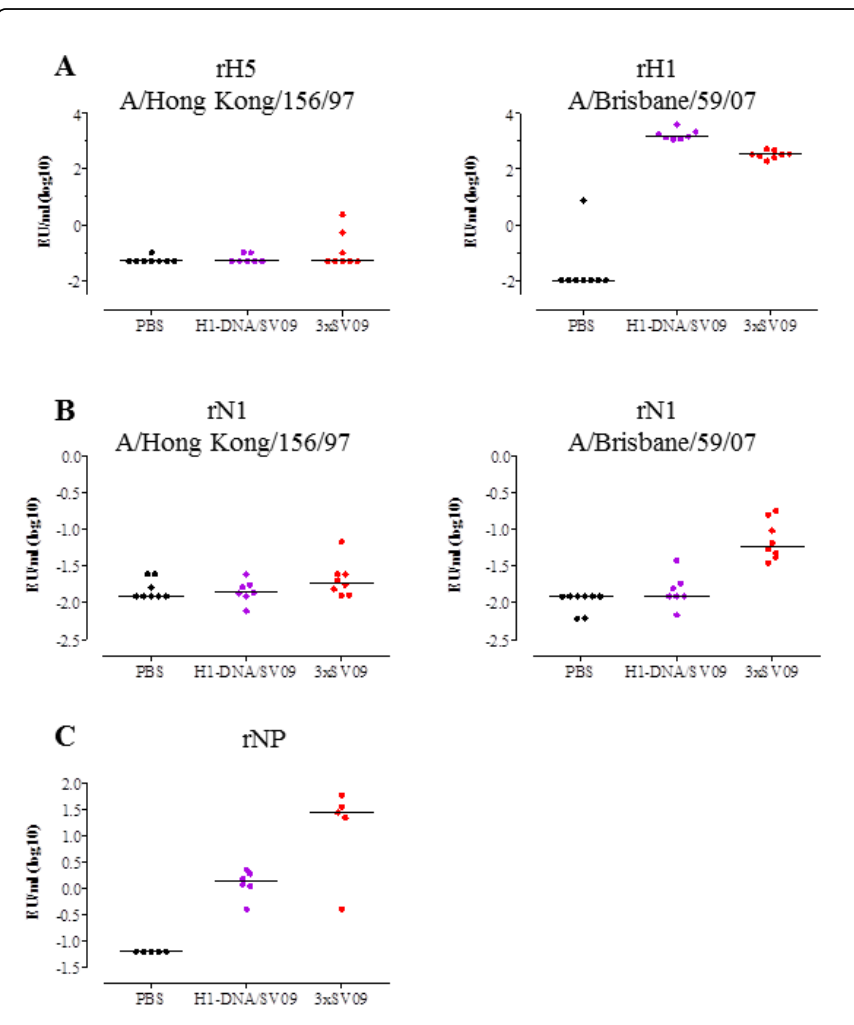

Figure 4: High titers of antibodies against NP, but not against H5 $\mathrm{HA}$ or NA, are elicited with both vaccination regimens and reflect observed difference in heterosubtypic protection. Characterization of humoral immune response induced by 3x Inflexal 2009 (3xSV09), 3x H1 HA DNA followed by a boost of Inflexal 2009 (H1DNA/SV09) or $4 \mathrm{x}$ PBS (PBS) in pre-challenge serum of mice challenged with H5N1 A/Hong Kong/156/97 (Immunogenicity of animals in challenge is reported; $n=8$ for PBS and 3x SV09; $n=7$ for H1-DNA/SV09). Binding Ab titer measured in ELISA against A) rHA of H5N1 A/Hong Kong/156/97 and rHA of H1N1 A/Brisbane/ 59/07 B) rNA of H5N1 A/Hong Kong/156/97 and rNA of H1N1 A/ Brisbane/59/07 C) rNP of A/Puerto Rico/8/34 (due to lacking serum $\mathrm{n}=5$ for PBS; $\mathrm{n}=5$ for $3 \mathrm{x}$ SV09; $\mathrm{n}=6$ for H1-DNA/SV09). Group medians are shown.

Since HA and NA specific assays did not appear to correlate with the observed protection against H5N1, we assessed the level of NP antibodies. As NP is highly conserved between influenza a group 1 virus (e.g., an amino acid homology above 90\%) we did not differentiate between vaccine homologous and challenge strain NP. Pre-challenge serum of mice immunized with H1-DNA/SV09 contained moderate level of anti-NP antibodies, and comparable to the level observed after single immunization with SV09 (data not shown) while the mean titer of anti-NP antibodies elicited with 3xSV09 was approximately 11.4-fold higher (Figure 4C).

\section{Discussion}

In this study we confirm the previous finding [30] that the breadth of heterologous protection elicited with seasonal influenza vaccine can be enhanced by priming the vaccine with DNA expressing vaccinecorresponding H1 HA. In addition, we demonstrate that this regimen is less efficient than multiple immunizations with seasonal vaccine in conferring protection against heterosubtypic H5N1. Among tested immune parameters only the anti-NP antibody titer followed the pattern of the protection between the two vaccination regimens.

Influenza specific antibodies can protect against influenza infection via a number of different immunological mechanisms. Neutralizing antibodies can prevent viral attachment to target cells $[37,38]$, inhibit release from endosomes $[22,37,39]$ or block egress[37,40]. Binding antibodies can mediate protection via indirect mechanisms such as antibody-dependent cellular cytotoxicity (ADCC) or antibodydependent complement mediated cytotoxicity (ADCMC) $[18,41]$. Influenza specific T-cells play a role in clearing infected cells and thereby limit the virus spread and host morbidity [4,42-44]. While vaccine- or infection-elicited protection against closely related viruses predominantly relies on hemagglutination-inhibiting antibodies that block the viral attachment to sialic acid receptors at cell surface, the protection against distant viruses is less well understood. It is likely that for different vaccines different mechanisms contribute to heterosubtypic protection, depending on the vaccine composition, formulation and schedule.

DNA plasmids as vectors for antigen delivery have been used in several fields to enhance the level and the breadth of the immune response [45-47]. The mechanism underlying the increased breadth of the humoral immune response induced when priming with DNA is suggested to be due to the increased number and diversity of induced CD4 $\mathrm{T}$ cells which can increase the expansion of antigen specific $\mathrm{B}$ cells $[45,48,49]$. In the influenza field, this strategy has been implemented primarily for pandemic $\mathrm{H} 5 \mathrm{~N} 1$ vaccines [50-52] but also in the development of cross-protective seasonal influenza vaccines $[30,35]$. In our hands, priming virosomal seasonal influenza vaccine with DNA expressing vaccine-corresponding H1 HA enhanced the heterologous protection in two different influenza challenge models, confirming the results from Wei et al., [30] for a split influenza vaccine. However, when evaluating the regimens' ability to confer heterosubtypic protection we found that priming the virosomal vaccine with H1-DNA did not improve the vaccines ability to confer H5N1 protection. Though eliciting a higher cross reactive HA specific $\mathrm{T}$ cell response than the homologous prime/boost regimen neither H1DNA/SV09 nor 3xSV09 elicited detectable cross reactive $\mathrm{HA} \mathrm{Ab}$ responses. Nevertheless, the homologous prime boost regimen, $3 \times$ SV09, was able to confer a higher level of survival after challenge with the heterosubtypic H5N1 strain.

We reported previously that vaccinating mice with SV11 according to the same homologous prime/boost regimen induced a comparable level of survival against H5N1. Unlike 3xSV09, the protection induced by3xSV11 correlated with the vaccine mediated H5 HA binding antibodies induced. The difference seen in protective capacity and immunogenicity between SV09 and SV11 is likely due to difference in vaccine composition. These results further emphasize the difficulty in predicting the cross protective capacity of a seasonal influenza vaccine based solely on immunogenicity.

In the current study, T-cell immunity was elicited only with H1DNA priming, so the superior protection against $\mathrm{H} 5 \mathrm{~N} 1$ by homologous vaccination could not be explained by HA-directed cellular immunity. Though we cannot rule out the level of cellular immunity against NA, our historical data indicate that these seasonal influenza vaccines do not induce NA-directed T-cell immunity. In combination with a lack of HA and NA cross-reactive antibodies, these findings suggest that responses to seasonal vaccine components other 
than HA or NA may contribute to the heterosubtypic $\mathrm{H} 5 \mathrm{~N} 1$ protection. Highly conserved influenza core proteins, such as nucleoprotein (NP), have frequently been investigated for their ability to induce cross-protection $[5,7,53,54]$. While primary mechanism of protection was considered to be via CD8 effector cells, a role for anti$\mathrm{NP}$ antibodies has recently been suggested [55,56]. Vaccine candidates containing NP have recently been shown to induce cross-protection in mice, and passive transfer experiments of anti-NP IgG have confirmed the ability of NP antibodies to confer protection [55-57]. Here we demonstrate that anti-NP antibodies are elicited by both vaccination regimens and it is possible that they contribute to the heterosubtypic (H5N1) protection conferred with vaccination modules comprised of SV09. The anti-NP titer induced with the heterologous prime/boost vaccine (H1-DNA/SV), the regimen that conferred weaker protection against $\mathrm{H} 5 \mathrm{~N} 1$, was lower and comparable to the titer level elicited with a single vaccination with SV (data not shown). This was as expected considering that DNA used for priming encodes only HA and not NP.

As we did not measure NP-specific T cells, we cannot formally prove that they do not play a role in the heterosubtypic protection elicited with 3xSV09. However, considering that 3xSV09 was not very efficient in eliciting HA-specific $\mathrm{T}$ cells, and our historical data indicating that this type of vaccines does not induce strong NP-specific $\mathrm{T}$ cells (data not shown), we consider it unlikely that NP T cells play a significant role for 3xSV09 vaccine-mediated heterosubtypic protection. It is also unlikely that NP-specific T cells played a role in protection elicited with H1-DNA/SV09 considering that the DNA encoded only HA and not NP.

The exact mechanism by which NP-specific antibodies mediate protection is not completely understood. In recent studies it has been established that NP is presented on the surface of virus-infected cells during budding of new viruses $[58,59]$ and the mechanism by which the anti-NP antibodies mediate protection has been shown to be $\mathrm{Fc} \gamma \mathrm{R}$ dependent [57]. Furthermore, Jegaskanda et al., [60] have shown, using NK-cell activation assay, that a trivalent inactivated influenza vaccine induce NP-mediated ADCC responses. Thus, it is possible that antibodies directed against influenza NP contribute to the heterosubtypic $\mathrm{H} 5 \mathrm{~N} 1$ protection observed in our study through these mechanisms.

In conclusion, we demonstrate that priming a seasonal influenza vaccine with HA encoding DNA and thereby improve its cross reactive HA T-cell response does not improve its ability to cross protect against H5N1 influenza virus. Despite the lack of detectable cross reactive HA and $\mathrm{NA} \mathrm{Ab}$ titers a homologous prime /boost regimen of the vaccine improved its ability to confer cross protection against H5N1. While HA and NA immunogenicity appears to play a minor role, NP-binding antibodies are the only immunogenicity parameter tested, which follows the same pattern as the heterosubtypic protection between the two studied vaccination regimens. We suggest that adding NP encoding DNA in the combination vaccine schedule may be an interesting approach to further broaden the protective activity of this heterologous prime/boost vaccine regimen in future studies.

\section{References}

1. WHO (2015) WHO fact sheet.

2. Gerdil C (2003) The annual production cycle for influenza vaccine. Vaccine 21: 1776-1779.

3. Pica N, Palese P (2013) Toward a universal influenza virus vaccine: prospects and challenges. Annu Rev Med 64: 189-202.
4. Hillaire ML, Osterhaus AD, Rimmelzwaan GF (2011) Induction of virusspecific cytotoxic T lymphocytes as a basis for the development of broadly protective influenza vaccines. J Biomed Biotechnol.

5. Epstein SL, Kong WP, Misplon JA, Lo CY, Tumpey TM, et al. (2005) Protection against multiple influenza A subtypes by vaccination with highly conserved nucleoprotein. Vaccine 23: 5404-5410.

6. Price GE, Soboleski MR, Lo CY, Misplon JA, Pappas C, et al. (2009) Vaccination focusing immunity on conserved antigens protects mice and ferrets against virulent $\mathrm{H} 1 \mathrm{~N} 1$ and $\mathrm{H} 5 \mathrm{~N} 1$ influenza A viruses. Vaccine 27: 6512-6521.

7. Hessel A, Dacho SH, Coulibaly S, Portsmouth D, Kreil TR, et al. (2014) MVA vectors expressing conserved influenza proteins protect mice against lethal challenge with $\mathrm{H} 5 \mathrm{~N} 1, \mathrm{H} 9 \mathrm{~N} 2$ and $\mathrm{H} 7 \mathrm{~N} 1$ viruses. PLoS One 9: e88340.

8. Sui Z, Chen Q, Fang F, Zheng M, Chen Z (2010) Cross-protection against influenza virus infection by intranasal administration of M1-based vaccine with chitosan as an adjuvant. Vaccine 28: 7690-7698.

9. Margine I, Krammer F, Hai R, Heaton NS, Tan GS, et al. (2013) Hemagglutinin stalk-based universal vaccine constructs protect against group 2 influenza A viruses. J Virol 87: 10435-10446.

10. Wang TT, Tan GS, Hai R, Pica N, Ngai L, et al. (2010) Vaccination with a synthetic peptide from the influenza virus hemagglutinin provides protection against distinct viral subtypes. Proc Natl Acad Sci U S A 107: 18979-18984.

11. Steel J, Lowen AC, Wang TT, Yondola M, Gao Q, et al. (2010) Influenza virus vaccine based on the conserved hemagglutinin stalk domain. MBio 1.

12. Impagliazzo A Milder F, Kuipers H, Wagner MV, Zhu X, et al. (2015) A stable trimeric influenza hemagglutinin stem as a broadly protective immunogen. Science 349: 1301-1306.

13. Yassine HM, Boyington JC, McTamney PM, Wei CJ, Kanekiyo M, et al. (2015) Hemagglutinin-stem nanoparticles generate heterosubtypic influenza protection. Nat Med 21: 1065-1070.

14. Easterbrook JD, Schwartzman LM, Gao J, Kash JC, Morens DM, et al (2012) Protection against a lethal H5N1 influenza challenge by intranasal immunization with virus-like particles containing 2009 pandemic H1N1 neuraminidase in mice. Virology 432: 39-44.

15. Sandbulte MR, Jimenez GS, Boon AC, Smith LR, Treanor JJ, et al. (2007) Cross-reactive neuraminidase antibodies afford partial protection against $\mathrm{H} 5 \mathrm{~N} 1$ in mice and are present in unexposed humans. PLoS medicine 4: e59.

16. Neirynck S1, Deroo T, Saelens X, Vanlandschoot P, Jou WM, et al. (1999) A universal influenza A vaccine based on the extracellular domain of the M2 protein. Nat Med 5: 1157-1163.

17. Song JM, Van Rooijen N, Bozja J, Compans RW, Kang SM (2011) Vaccination inducing broad and improved cross protection against multiple subtypes of influenza A virus. Proceedings of the National Academy of Sciences of the United States of America 108: 757-761.

18. El Bakkouri K, Descamps F, De Filette M, Smet A, Festjens E, et al. (2011) Universal vaccine based on ectodomain of matrix protein 2 of influenza A: Fc receptors and alveolar macrophages mediate protection. J Immunol 186: 1022-1031.

19. Ekiert DC, Bhabha G, Elsliger MA, Friesen RH, Jongeneelen M, et al. (2009) Antibody recognition of a highly conserved influenza virus epitope. Science 324: 246-251.

20. Ekiert DC, Friesen RH, Bhabha G, Kwaks T, Jongeneelen M, et al. (2011) A highly conserved neutralizing epitope on group 2 influenza A viruses. Science 333: 843-850.

21. Throsby M, van den Brink E, Jongeneelen M, Poon LL, Alard P, et al. (2008) Heterosubtypic neutralizing monoclonal antibodies crossprotective against $\mathrm{H} 5 \mathrm{~N} 1$ and $\mathrm{H} 1 \mathrm{~N} 1$ recovered from human IgM+ memory B cells. PloS one 3: e3942.

22. Dreyfus C, Laursen NS, Kwaks T, Zuijdgeest D, Khayat R, et al. (2012) Highly conserved protective epitopes on influenza B viruses. Science 337: 1343-1348. 
23. Wan H, Gao J, Xu K, Chen H, Couzens LK, et al. (2013) Molecular basis for broad neuraminidase immunity: conserved epitopes in seasonal and pandemic H1N1 as well as H5N1 influenza viruses. J Virol 87: 9290-9300.

24. Gravel C, Li C, Wang J, Hashem AM, Jaentschke B, et al. (2010) Qualitative and quantitative analyses of virtually all subtypes of influenza $\mathrm{A}$ and $\mathrm{B}$ viral neuraminidases using antibodies targeting the universally conserved sequences. Vaccine 28: 5774-5784.

25. Corti D1, Voss J, Gamblin SJ, Codoni G, Macagno A, et al. (2011) A neutralizing antibody selected from plasma cells that binds to group 1 and group 2 influenza A hemagglutinins. Science 333: 850-856.

26. Gioia C, Castilletti C, Tempestilli M, Piacentini P, Bordi L, et al. (2008) Cross-subtype immunity against avian influenza in persons recently vaccinated for influenza. Emerg Infect Dis 14: 121-128.

27. Tanimoto T, Nakatsu R, Fuke I, Ishikawa T, Ishibashi M, et al. (2005) Estimation of the neuraminidase content of influenza viruses and splitproduct vaccines by immunochromatography. Vaccine 23: 4598-4609.

28. Co MD, Orphin L, Cruz J, Pazoles P, Green KM, et al. (2009) In vitro evidence that commercial influenza vaccines are not similar in their ability to activate human T cell responses. Vaccine 27: 319-327.

29. van Maurik A, Sabarth N, Dacho HS, Bruhl P, Schwendinger M, et al. (2010) Seasonal influenza vaccine elicits heterosubtypic immunity against $\mathrm{H} 5 \mathrm{~N} 1$ that can be further boosted by $\mathrm{H} 5 \mathrm{~N} 1$ vaccination. Vaccine 28 1778-1785.

30. Wei CJ, Boyington JC, McTamney PM, Kong WP, Pearce MB, et al. (2010) Induction of broadly neutralizing $\mathrm{H} 1 \mathrm{~N} 1$ influenza antibodies by vaccination. Science 329: 1060-1064

31. Ichinohe T, Tamura S, Kawaguchi A, Ninomiya A, Imai M, et al. (2007) Cross-protection against $\mathrm{H} 5 \mathrm{~N} 1$ influenza virus infection is afforded by intranasal inoculation with seasonal trivalent inactivated influenza vaccine. The Journal of infectious diseases 196: 1313-1320.

32. Roos A, Roozendaal R, Theeuwsen J, Riahi S, Vaneman J, et al. (2015) Protection against $\mathrm{H} 5 \mathrm{~N} 1$ by multiple immunizations with seasonal influenza vaccine in mice is correlated with $\mathrm{H} 5$ cross-reactive antibodies. Vaccine 33: 1739-1747.

33. Rockman S, Brown LE, Barr IG, Gilbertson B, Lowther S, et al. (2013) Neuraminidase-inhibiting antibody is a correlate of cross-protection against lethal $\mathrm{H} 5 \mathrm{~N} 1$ influenza virus in ferrets immunized with seasonal influenza vaccine. J Virol 87: 3053-3061.

34. Sambhara S, Kurichh A, Miranda R, Tumpey T, Rowe T, et al. (2001) Heterosubtypic immunity against human influenza A viruses, including recently emerged avian $\mathrm{H} 5$ and $\mathrm{H} 9$ viruses, induced by FLU-ISCOM vaccine in mice requires both cytotoxic T-lymphocyte and macrophage function. Cell Immunol 211: 143-153.

35. A service of the U.S. National Institutes of Health (2009) VRC 307: A D/B Randomized $\mathrm{Ph}$. I Study of Safety/Immunogenicity of a Prime-Boost Schedule of an Investigational DNA Influenza Vaccine, Followed by the Seasonal Influenza Trivalent Inactivated Vaccine (TIV), Compared to TIV Alone in Healthy Adults. 7 ed. Bethesda National Institutes of Health Clinical Center, USA.

36. RadoseviÄ $\ddagger$ K, Rodriguez A, Mintardjo R, Tax D, Bengtsson KL, et al. (2008) Antibody and T-cell responses to a virosomal adjuvanted H9N2 avian influenza vaccine: impact of distinct additional adjuvants. Vaccine 26: 3640-3646.

37. Brandenburg B, Koudstaal W, Goudsmit J, Klaren V, Tang C, et al. (2013) Mechanisms of hemagglutinin targeted influenza virus neutralization. PLoS One 8: e80034.

38. Skehel JJ, Wiley DC (2000) Receptor binding and membrane fusion in virus entry: the influenza hemagglutinin. Annu Rev Biochem 69: 531-569.

39. Hashem AM, Van Domselaar G, Li C, Wang J, She YM, et al. (2010) Universal antibodies against the highly conserved influenza fusion peptide cross-neutralize several subtypes of influenza A virus. Biochem Biophys Res Commun 403: 247-251.

40. Marcelin G, Sandbulte MR, Webby RJ (2012) Contribution of antibody production against neuraminidase to the protection afforded by influenza vaccines. Rev Med Virol 22: 267-279.
41. Jegaskanda S, Job ER, Kramski M, Laurie K, Isitman G, et al. (2013) Cross-reactive influenza-specific antibody-dependent cellular cytotoxicity antibodies in the absence of neutralizing antibodies. J Immunol 190 1837-1848.

42. Wilkinson TM, Li CK, Chui CS, Huang AK, Perkins M, et al. (2012) Preexisting influenza-specific CD4+ $\mathrm{T}$ cells correlate with disease protection against influenza challenge in humans. Nat Med 18: 274-280.

43. McMichael AJ, Gotch FM, Noble GR, Beare PA (1983) Cytotoxic T-cell immunity to influenza. N Engl J Med 309: 13-17.

44. Baz M, Boonnak K, Paskel M, Santos C, Powell T, et al. (2015) Nonreplicating Influenza A Virus Vaccines Confer Broad Protection against Lethal Challenge. MBio 6.

45. Wu L, Kong WP, Nabel GJ (2005) Enhanced breadth of CD4 T-cell immunity by DNA prime and adenovirus boost immunization to human immunodeficiency virus Env and Gag immunogens. J Virol 79: 8024-8031.

46. Schneider J, Gilbert SC, Blanchard TJ, Hanke T, Robson KJ, et al. (1998) Enhanced immunogenicity for CD8 $+\mathrm{T}$ cell induction and complete protective efficacy of malaria DNA vaccination by boosting with modified vaccinia virus Ankara. Nat Med 4: 397-402.

47. Shedlock DJ, Aviles J, Talbott KT, Wong G, Wu SJ, et al. (2013) Induction of broad cytotoxic T cells by protective DNA vaccination against Marburg and Ebola. Mol Ther 21: 1432-1444.

48. Khurana S, Wu J, Dimitrova M, King LR, Manischewitz J, et al. (2013) DNA priming prior to inactivated influenza $\mathrm{A}(\mathrm{H} 5 \mathrm{~N} 1)$ vaccination expands the antibody epitope repertoire and increases affinity maturation in a boost-interval-dependent manner in adults. J Infect Dis 208 : 413-417.

49. Liu MA (2010) Immunologic basis of vaccine vectors. Immunity 33 504-515.

50. Wang G, Zhou F, Buchy P, Zuo T, Hu H, et al. (2014) DNA prime and virus-like particle boost from a single $\mathrm{H} 5 \mathrm{~N} 1$ strain elicits broadly neutralizing antibody responses against head region of $\mathrm{H} 5$ hemagglutinin. J Infect Dis 209: 676-685.

51. Ledgerwood JE, Wei CJ, Hu Z, Gordon IJ, Enama ME, et al. (2011) DNA priming and influenza vaccine immunogenicity: two phase 1 open label randomised clinical trials. Lancet Infect Dis 11: 916-924.

52. Ding H, Tsai C, Gutiérrez RA, Zhou F, Buchy P, et al. (2011) Superior neutralizing antibody response and protection in mice vaccinated with heterologous DNA prime and virus like particle boost against HPAI H5N1 virus. PLoS One 6: e16563.

53. Ulmer JB, Donnelly JJ, Parker SE, Rhodes GH, Felgner PL, et al. (1993) Heterologous protection against influenza by injection of DNA encoding a viral protein. Science 259: 1745-1749.

54. Lo CY, Wu Z, Misplon JA, Price GE, Pappas C, et al. (2008) Comparison of vaccines for induction of heterosubtypic immunity to influenza $A$ virus: cold-adapted vaccine versus DNA prime-adenovirus boost strategies. Vaccine 26: 2062-2072.

55. Carragher DM, Kaminski DA, Moquin A, Hartson L, Randall TD (2008) A novel role for non-neutralizing antibodies against nucleoprotein in facilitating resistance to influenza virus. J Immunol 181: 4168-4176.

56. Lamere MW, Moquin A, Lee FE, Misra RS, Blair PJ, et al. (2011) Regulation of antinucleoprotein IgG by systemic vaccination and its effect on influenza virus clearance. J Virol 85: 5027-5035.

57. LaMere MW, Lam HT, Moquin A, Haynes L, Lund FE, et al. (2011) Contributions of antinucleoprotein IgG to heterosubtypic immunity against influenza virus. J Immunol 186: 4331-4339.

58. Bodewes R, Geelhoed-Mieras MM, Wrammert J, Ahmed R, Wilson PC, et al. (2013) In vitro assessment of the immunological significance of a human monoclonal antibody directed to the influenza a virus nucleoprotein. Clin Vaccine Immunol 20: 1333-1337.

59. Nayak DP, Balogun RA, Yamada H, Zhou ZH, Barman S (2009) Influenza virus morphogenesis and budding. Virus Res 143: 147-161.

60. Jegaskanda S, Amarasena TH, Laurie KL, Tan HX, Butler J, et al. (2013) Standard trivalent influenza virus protein vaccination does not prime 
Citation: Roos A, Roozendaal R, Riahi SD, Vreugdenhil J, Vaneman J, et al. (2015) H5N1 Protection by Seasonal Influenza Vaccine in Homologous and Heterologous Prime/Boost Vaccination. J Vaccines Vaccin 6: 299. doi:2157-7560.1000299

Page 9 of 9

antibody-dependent cellular cytotoxicity in macaques. J Virol 87: 13706-13718. 